\title{
Infrared Thermography as a Method of Verification in Raynaud's Phenomenon
}

\author{
Lotte Lindberg ${ }^{1, *(\mathbb{D}}$, Bent Kristensen ${ }^{1}$, Ebbe Eldrup ${ }^{2}$, Jane Frølund Thomsen ${ }^{3}$ and Lars Thorbjørn Jensen ${ }^{1}$ \\ 1 Department of Nuclear Medicine, Copenhagen University Hospital, Herlev Hospital, \\ Borgmester Ib Juuls Vej 1, 2730 Herlev, Denmark; bent.kristensen.01@regionh.dk (B.K.); \\ lars.thorbjoern.jensen@regionh.dk (L.T.J.) \\ 2 Department of Endocrinology, Copenhagen University Hospital, Herlev Hospital, Borgmester Ib Juuls Vej 1, \\ 2730 Herlev, Denmark; ebbe.eldrup@regionh.dk \\ 3 Department of Occupational and Environmental Medicine, Copenhagen University Hospital, Bispebjerg and \\ Frederiksberg Hospital, Bispebjerg Bakke 23, 2400 Copenhagen, Denmark; jane.froelund.thomsen@regionh.dk \\ * Correspondence: lotte.lindberg.01@regionh.dk
}

Citation: Lindberg, L.; Kristensen, B. Eldrup, E.; Thomsen, J.F.; Jensen, L.T. Infrared Thermography as a Method of Verification in Raynaud's Phenomenon. Diagnostics 2021, 11, 981. https://doi.org/10.3390/ diagnostics11060981

Academic Editor: Malek Makki

Received: 13 April 2021

Accepted: 21 May 2021

Published: 28 May 2021

Publisher's Note: MDPI stays neutral with regard to jurisdictional claims in published maps and institutional affiliations.

Copyright: (c) 2021 by the authors. Licensee MDPI, Basel, Switzerland. This article is an open access article distributed under the terms and conditions of the Creative Commons Attribution (CC BY) license (https:// creativecommons.org/licenses/by/ $4.0 /)$.

\begin{abstract}
Raynaud's phenomenon (RP) is characterized by the episodic whitening of the fingers upon exposure to cold. A recently described thermographic algorithm was proposed as a diagnostic replacement of the currently applied finger systolic pressure (FSP) test. The aim of the study was to evaluate the performance of the thermographic algorithm when applied in patients suspected of having RP. Forty-three patients were examined using thermographic imaging after local cooling of the hands in water of $10^{\circ} \mathrm{C}$ for $1 \mathrm{~min}$. The thermographic algorithm was applied to predict the probability of RP. The performance of the algorithm was evaluated with different cut-off levels. A new algorithm was proposed based on patients from the target population. The performance of the tested algorithm was noninferior to the FSP test, when a cut-off level of 0.05 was applied, yielding a sensitivity and specificity of $69 \%$ and $58 \%$, respectively. The accuracy was $66 \%$. The FSP test had a sensitivity and specificity of $77 \%$ and $37 \%$, respectively, and the accuracy was $59 \%$. The thermographic method proved useful for detecting RP and was able to replace the FSP test as a diagnostic test. The alternative algorithm revealed that other thermographic variables were more predictive of the target population, but this should be verified in future patients.
\end{abstract}

Keywords: cold challenge; diagnostic method; infrared thermographic imaging; secondary Raynaud's phenomenon; vibration white finger

\section{Introduction}

Raynaud's phenomenon (RP) is characterized by the episodic whitening of the fingers upon exposure to cold. Although the cause cannot be identified in most patients, RP can be induced by certain drugs, an underlying rheumatic disease, or repeated exposure to handarm vibrations [1]. RP is typically a clinical diagnosis based on medical history, a physical examination, and blood tests. However, RP symptoms are not always characteristic. In vibration white finger (VWF), which is caused by occupational exposure to vibration, the diagnosis must be verified in order for the condition to be accepted as an occupational injury. Furthermore, a correct diagnosis is important for the patient, as continued exposure may aggravate the disease [2], possibly resulting in recommendations to change professions. The diagnosis is verified if an episode is witnessed by a physician or the patient is able to capture a photo of an episode, either of which has proven easy to obtain. As a result, an objective examination that includes a cold provocation test is often required to substantiate the diagnosis.

The method of systolic blood pressure with cold provocation is currently applied in our department and has been used for decades [3]. Under this method, the decrease in finger systolic blood pressure is measured after $10 \mathrm{~min}$ of total body cold exposure and 
local finger cooling. This method is cumbersome, time-consuming, and unpleasant for the patient. Moreover, despite obvious anamnestic signs of RP, the method sometimes neglects to detect the disease. Additionally, the method requires outdated technical equipment no longer supported by the manufacturer, which makes it necessary to develop a new procedure for detecting RP.

Infrared thermographic imaging has been the subject of numerous investigations that include various patient groups, purposes, and outcomes. The performances of the different methods vary widely and seem to depend on the prevalence of RP in the study population, the severity of the symptoms [4,5], and the possibility of closely controlling the environmental conditions during the examination $[4,6,7]$. We recently investigated infrared thermographic imaging as a method for distinguishing patients with primary Raynaud's phenomenon (pRP) from healthy individuals [8]. The study generated a prediction model that was able to predict the presence or absence of RP with a sensitivity of $82 \%$ and a negative predictive value (NPV) of $93 \%$ and was not inferior to the previous studies on thermographic imaging [5,9-11]. Thus, infrared thermographic imaging showed great promise as a verification method in patients with $\mathrm{RP}$, where confirmation of the diagnosis is needed.

The aim of the present study was to test the newly described thermographic method in patients with RP, primarily associated with occupational exposure to vibration and whose diagnosis was not yet verified, and compare the performance of the method with that of the currently applied method. The hypothesis was that the new method would not be inferior to the finger systolic pressure (FSP) test in performance and able to replace this method as a diagnostic test. In addition, we wanted to explore whether the thermographic parameters characteristic for $\mathrm{PRP}$ and VWF, respectively, were similar.

\section{Materials and Methods}

\subsection{Participants}

Forty-three patients with suspected RP were included in the study. Thirty-five patients were referred from two Departments of Occupational and Environmental Medicine in Eastern Denmark due to occupational exposure to vibration and suspected VWF. The remaining eight patients were referred from other medical specialties, including general practitioners, and had not been exposed to vibration. Thus, the origin of RP was unknown for this subgroup. None of the patients had diabetes mellitus, were previously treated with chemotherapy, or were diagnosed with vitamin $B_{12}$ deficiency or renal impairment. Twenty-two patients had already been examined using the existing diagnostic method (FSP test) from January 2014 to October 2016. The remaining 21 patients were recruited immediately after an examination with the FSP test (from January 2017 to January 2019). The two groups of patients were named the retrospective and the prospective groups, respectively, and were included consecutively.

For the purpose of evaluating the performance of the algorithm in the patients suspected of RP, the clinical diagnosis was used as the reference standard. Thus, the clinical diagnosis was made by two senior consultants upon review of the medical records of 40 patients (three were missing). The two readers independently assigned a diagnosis (positive or negative of RP) to each patient blinded to the results of the FSP and thermographic tests. Eight diagnostic disagreements between the readers were discussed, and joint diagnoses were made. The decision, which was based solely on the symptoms, physical examination, and blood test results described in the medical records, served as the true diagnosis of RP. The medical records of three patients were missing.

The control group was identical to the control group described in reference [8]. The healthy participants were recruited through advertisements in Herlev and Gentofte Hospital and in local newspapers. All 58 participants included in the control group had no symptoms of RP or history of occupational exposure to vibration. Moreover, they had no medical record of chronic illnesses or health issues affecting the blood vessels, especially heart or lung diseases, or connective tissue diseases. They did not have vitamin $\mathrm{B}_{12}$ defi- 
ciency or alcohol abuse, they were alleged nonsmokers (one participant changed smoking status after inclusion), and they did not take prescription drugs. Thus, 101 subjects were examined using the thermographic method.

Blood tests were taken in order to exclude differential diagnoses to RP: folic acid, vitamin $\mathrm{B}_{12}$, hemoglobin, albumin, ionized calcium, potassium, magnesium, sodium, glucose, $\mathrm{HbA1c}, \mathrm{TSH}, \mathrm{CRP}$, and vitamin $\mathrm{D}$. The examinations were performed at the Department of Nuclear Medicine, Copenhagen University Hospital, Herlev Hospital, from January 2017 to February 2019.

\subsection{Thermographic Imaging Procedure and Data Analysis}

The thermographic procedure, preparations, and subsequent analysis were conducted as previously described [8]. In short, the participants' hands were immersed in water of $10^{\circ} \mathrm{C}$ for $60 \mathrm{~s}$, and the rewarming period of the fingers was recorded using an infrared thermographic camera (FLIR SC600, FLIR ${ }^{\circledR}$ Systems, Wilsonville, OR, USA). The thermographic recordings resulted in rewarming curves, which were analyzed to obtain the seven temperature variables previously described [8]: baseline finger temperature $\left(t_{\text {base }}\right)$, finger temperature immediately after cooling $\left(\mathrm{t}_{0}\right)$, curve type, finger temperature halfway through rewarming $\left(t_{50}\right)$, end temperature $\left(t_{\text {end }}\right)$, time to $t_{\text {end }}$, and the percentage of temperature recovery at $t_{\text {end }}(\mathrm{R} \%)(\mathrm{R} \%=$ temperature increase/initial temperature decrease $) \times 100 \%$ [12]. The categorical variable curve type described the course of the temperature curve as either S-shaped or horizontal. See Figure 1 for an example of each curve type with the associated thermographic images at the essential time points.

\subsection{Finger Systolic Pressure (FSP) Test}

The currently applied FSP procedure uses body cooling, as well as local cooling, to $30{ }^{\circ} \mathrm{C}$ and $10^{\circ} \mathrm{C}$ to provoke an episode of RP. At $10{ }^{\circ} \mathrm{C}$, the discriminating threshold for the diagnosis of RP is defined as an FSP of $20 \%$ or less of the FSP at $30^{\circ} \mathrm{C}$, while an abnormal cold response is defined as an FSP of $20-63 \%$ of the FSP at $30^{\circ} \mathrm{C}$. An FSP above $63 \%$ of the FSP at $30{ }^{\circ} \mathrm{C}$ is defined as a normal response.

\subsection{Statistics}

Descriptive statistics were presented as the mean and standard deviation or median and interquartile range (IQR) where appropriate. Counts were presented as numbers and proportions. For normally distributed variables, group comparisons were done using the Student's $t$-test. For non-normally distributed variables, comparisons were done using the Wilcoxon rank-sum test. Due to the small cell counts, categorical variables were compared groupwise by applying Fisher's exact test.

The coefficients of the predictor variables (time to $t_{\text {end }}$ and $t_{\text {base }}$ ) (Table 1) and the respective values of time to $t_{\text {end }}$ and $t_{\text {base }}$ generated from the thermographic examination of the patients suspected of RP were used to calculate the patients' respective predicted probabilities of having RP. Different cut-off values of the predicted probability were assessed, including the cut-off value originally chosen. The performances of the thermographic algorithm and the FSP test were compared using superiority and noninferiority analyses, where a margin of $\Delta=-15 \%$ was chosen.

Table 1. The prediction model generated from 22 patients with pRP. Reproduced from reference [8]. The time to $t_{\mathrm{end}}=$ time to end temperature. The $t_{\mathrm{base}}=$ baseline finger temperature.

\begin{tabular}{ccccc}
\hline Predictor & Estimate & Std. Error & Wald $\chi^{2}$ & $p$-Value \\
\hline Intercept & 2.4 & 4.9 & 0.50 & 0.62 \\
time to tend & 0.11 & 0.04 & 2.9 & 0.004 \\
tbase & -0.30 & 0.15 & -2.0 & 0.04 \\
\hline
\end{tabular}



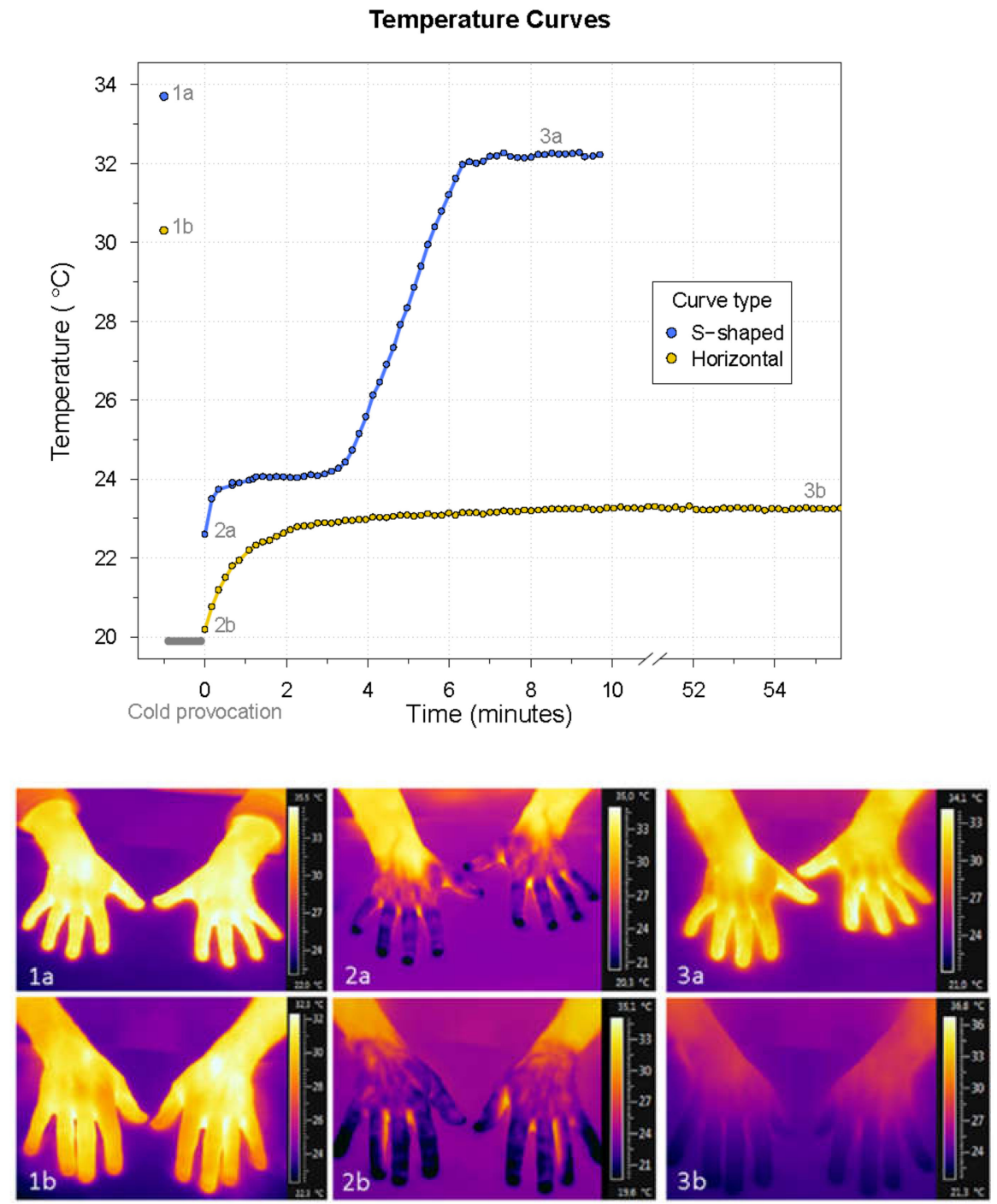

Figure 1. The two curve types identified during the analysis of the thermographic temperature curves. The numbers 1-3 refer to the time points of the baseline, immediately after cooling, and at the end of the thermographic examination, respectively. These time points correspond to the temperature variables baseline finger temperature $\left(t_{\text {base }}\right)$, finger temperature immediately after cooling $\left(t_{\text {nul }}\right)$, and end temperature $\left(t_{\text {end }}\right)$, respectively. The thermographic images associated with the mentioned time points are shown below the curves. The top bar shows the hands of a healthy individual (a), while the bottom bar presents the hands of a patient with RP (b). This figure is reproduced from the study, describing the recently developed algorithm [8].

After the evaluation of the previously developed thermographic method, a new algorithm was generated to investigate if $\mathrm{pRP}$ and VWF share the same thermographic characteristics. The fitting of a new logistic prediction model based on the thermographic parameters of the patients positive for RP in the present cohort and the healthy controls and evaluation of the model were conducted as described previously [8]. All the statistical calculations were done using the statistical software R, version 4.0.2, June 2020), (R Core Team (2020). R: A language and environment for statistical computing. R Foundation for Statistical Computing, Vienna, Austria. URL https:/ /www.R-project.org/), and the following packages: car, ClusterRankTest, confinterpret, growthrates, Hmisc, pROC, PropCIs, rms, and tableone. 


\section{Results}

\subsection{Clinical Characteristics}

Two patients and one healthy individual were excluded from the analyses, as their respective examinations ended prematurely (due to back pain and technical problems). Accordingly, the results from 98 subjects were included in the analyses. The participants consisted of $27(27.6 \%)$ women and $71(72.4 \%)$ men. Their clinical characteristics are presented in Table 2 .

Table 2. Clinical characteristics of the participants. Data are presented as number (\%), mean (SD), or median (IQR). Raynaud's phenomenon (RP).

\begin{tabular}{|c|c|c|c|c|}
\hline & & $\begin{array}{c}\text { Patients Suspected of } \\
\operatorname{RP}(n=41)\end{array}$ & Control Group $(n=57)$ & $p$-Value \\
\hline \multirow[t]{2}{*}{ Gender } & Male & $38(92.7)$ & $33(57.9)$ & $<0.001$ \\
\hline & Female & $3(7.3)$ & $24(42.1)$ & \\
\hline Age (Years) & & $56.2(9.3)$ & $57.8(12.5)$ & 0.49 \\
\hline \multirow[t]{4}{*}{ Family History of RP } & None & $34(82.9)$ & $48(84.2)$ & 0.69 \\
\hline & $1^{\circ}$ Relative & $5(12.2)$ & $5(8.8)$ & \\
\hline & $2^{\circ}$ Relative & $1(2.4)$ & $0(0.0)$ & \\
\hline & Unknown & $1(2.4)$ & $4(7.0)$ & \\
\hline \multirow{3}{*}{ Smoking } & Never & $12(29.3)$ & $35(61.4)$ & $<0.001$ \\
\hline & Current & $13(31.7)$ & $1(1.8)$ & \\
\hline & Former & $16(39.0)$ & $21(36.8)$ & \\
\hline Smoking (Pack Years) & & $15(0 ; 40)$ & $0(0 ; 3)$ & $<0.001$ \\
\hline Alcohol (Units/Week) & & $9(2 ; 15)$ & $4(2 ; 7)$ & 0.01 \\
\hline \multirow[t]{6}{*}{ Occupation } & Construction & $31(75.6)$ & $6(10.5)$ & $<0.001$ \\
\hline & Teaching and Healthcare & $2(4.9)$ & $30(52.6)$ & \\
\hline & $\begin{array}{l}\text { Commerce and } \\
\text { Transportation }\end{array}$ & $3(7.3)$ & $5(8.8)$ & \\
\hline & Industry & $2(4.9)$ & $6(10.5)$ & \\
\hline & Finance & $0(0.0)$ & $7(12.3)$ & \\
\hline & Other & $3(7.3)$ & $3(5.3)$ & \\
\hline
\end{tabular}

As defined in the exclusion criteria, none of the healthy participants were repeatedly exposed to vibration, while $34(83 \%)$ of the patients were exposed to vibration in relation to their occupation. Two (5\%) patients were treated for RP with drugs. In both cases, the treatment was paused $24 \mathrm{~h}$ prior to examination.

The patients in the retrospective group had symptoms of RP significantly longer than the patients in the prospective group (median (IQR): 10 years $(8 ; 15)$ and 5 years $(3 ; 8)$, respectively). Furthermore, the retrospective group had a higher alcohol intake, as well as a larger proportion of patients currently smoking, and, thereby, a higher consumption of tobacco than the prospective group. Although the potassium level was within the normal limits, the level was slightly higher in the retrospective group than in the group of healthy individuals.

The blood tests showed no differences between the patients and the healthy individuals in terms of folic acid, albumin, ionized calcium, magnesium, sodium, glucose, $\mathrm{HbA} 1 \mathrm{c}$, $\mathrm{TSH}, \mathrm{CRP}$, and Vitamin D. The hemoglobin was significantly lower in the control group, which was probably due to the larger proportion of female participants in this group (42\%) compared with the patient group $(7 \%)$. Vitamin $B_{12}$ was significantly lower and potassium significantly higher in the patient group than in the control group. The mean plasma level of vitamin $B_{12}$ was above the lower reference limit ( $\left.>200 \mathrm{pmol} / \mathrm{L}\right)$. However, for five patients and one healthy individual, their levels of vitamin $B_{12}$ were lower than the commonly used reference limit.

Based on the clinical decisions, a total of 26 of the 38 patients $(68 \%)$ were diagnosed with RP, while the diagnosis was rejected in 12 patients (32\%). The 38 patients refer to the total number of patients $(n=43)$ without the two patients whose thermographic results 
were excluded from analysis and without the three patients with missing medical records. When the results from the FSP test for the patients whose medical records were missing were included, the overall prevalence was $71 \%$. The results of the FSP test were positive for RP in 17 (42\%) patients, negative in $19(46 \%)$ patients, and intermediate in $5(12 \%)$ patients. When the FSP results were compared with the clinical decisions, the diagnosis concurred in 21 patients but not in the remaining 17 patients; twelve patients with a negative FSP test result were found positive for RP based on the clinical diagnosis, whereas five patients with a positive or intermediate FSP result were found negative for RP based on the clinical diagnosis. At the 0.05 cut-off value, the thermographic diagnosis concurred with the clinical decision in 24 patients but not in the remaining 14 patients. None of the participants reported adverse events from the thermographic or the FSP procedures.

\subsection{The Tested Thermographic Algorithm}

The characteristics from the thermographic examination of the patients suspected of having RP, the subsequent application of the diagnostic algorithm, and the chosen cut-off value (0.46) —as was suggested when the algorithm was developed —resulted in a correctly confirmed diagnosis in six patients and a correctly rejected diagnosis in 12 patients, corresponding to the test performances presented in Table 3. Thus, the new thermographic algorithm was better at rejecting the diagnosis than the FSP test but was less able to identify patients with RP. Applying lower cut-off levels resulted in a similar or slightly higher accuracy for the thermographic algorithm compared with the FSP test, indicating that the thermographic method is not inferior to the FSP test in terms of diagnostic ability. The 0.05 cut-off level resulted in a slightly higher accuracy, lower sensitivity, and a considerably higher specificity for the thermographic algorithm compared with the FSP test (Table 3).

Table 3. Measures of the test performance for the finger systolic pressure (FSP) test and the evaluated thermographic algorithm with the clinical decision as the reference diagnosis $(n=38)$. The thermographic algorithm is represented by four different predicted probability cut-off levels with associated $95 \%$ confidence intervals. PPV $=$ positive predictive value. $\mathrm{NPV}=$ negative predictive value .

\begin{tabular}{cccccc}
\hline Method & & & Thermographic Algorithm & FSP Test \\
\hline Cut-Off & 0.05 & 0.10 & 0.20 & 0.46 & 0.63 \\
\hline Sensitivity & $65 \%(46-81 \%)$ & $54 \%(35-71 \%)$ & $50 \%(32-68 \%)$ & $19 \%(9-38 \%)$ & $54 \%(33-73 \%)$ \\
Specificity & $58 \%(32-81 \%)$ & $67 \%(39-86 \%)$ & $75 \%(47-91 \%)$ & $100 \%(76-100 \%)$ & $58 \%(28-85 \%)$ \\
PPV & $77 \%(57-90 \%)$ & $78 \%(55-91 \%)$ & $81 \%(57-93 \%)$ & $100 \%(57-100 \%)$ & $74 \%(57-86 \%)$ \\
NPV & $44 \%(23-67 \%)$ & $40 \%(22-61 \%)$ & $41 \%(23-61 \%)$ & $36 \%(22-53 \%)$ & $37 \%(24-52 \%)$ \\
Accuracy & $63 \%(47-77 \%)$ & $58 \%(42-72 \%)$ & $58 \%(42-72 \%)$ & $45 \%(30-60 \%)$ & $55 \%(38-71 \%)$ \\
\hline
\end{tabular}

When comparing the thermographic test performance (cut-off 0.05) with the FSP test performance, the thermographic test was not shown to be superior to the FSP test for any of the performance measures. However, the sensitivity and accuracy of the thermographic test were shown to be noninferior to that of the FSP test by a 15\% margin.

\subsection{A New Algorithm}

Based on the assumption that the verification of RP of a different origin requires alternative thermographic characteristics, a new algorithm was developed based on the patients diagnosed with RP by the clinical evaluation and the healthy controls.

In addition to the curve and temperature variables, age was also included in the analysis but did not contribute significantly to the prediction of RP. Gender was not included because of the small number of female patients. The binary response variable of the logistic regression model was the clinical diagnosis of RP assessed by two different senior consultants. According to the fitted logistic regression model, the combinations of predictors best able to predict the presence or absence of RP in the target population were $t_{50}$, curve type, and time to $t_{\text {end }}$. The model specifics are presented in Table 4. 
Table 4. The new prediction model based on the patients diagnosed with Raynaud's phenomenon by the clinical evaluation $(n=26)$ and the healthy controls $(n=57)$. Estimates are the respective coefficients of the model with the associated standard errors. The $p$-values show the predictors' respective contributions to the model.

\begin{tabular}{ccccc}
\hline Predictor & Estimate & Std. Error & Wald $\chi^{2}$ & $p$-Value \\
\hline Intercept & -28.9 & 8.0 & -3.6 & $<0.001$ \\
$t_{50}$ & 0.92 & 0.27 & 3.4 & $<0.001$ \\
curve type & 4.8 & 1.4 & 3.3 & $<0.001$ \\
${\text { time to } t_{\text {end }}}$ & 0.06 & 0.02 & 2.7 & 0.007 \\
\hline
\end{tabular}

The diagnostics revealed no significant deviations from the model assumptions. Five outliers (patients, as well as controls) were identified and reviewed. As no irregular values were found, they were all included in the analyses. The calibration plot and the calibration test showed that the intercept $(=-0.07)$ and the slope $(0.84)$ did not differ significantly from the ideal line $(p=0.20)$, indicating that the predicted probability equaled the actual (observed) probability. The AUC was 0.86 (95\% confidence interval: $0.78-0.94$ ) and the Brier score 0.17 after bootstrapping. The Brier score is an evaluation score of the logistic regression models. With values from 0 to 1 , a low Brier score indicates well-calibrated predictions.

\subsection{Cut-Off Level}

Similar to the first thermographic algorithm, the new algorithm conveyed a predicted probability of having RP. The ability of the method to identify patients who have RP and the ability to correctly reject the diagnosis will, to some extent, depend on the chosen cut-off level. This is illustrated in Table 3, where the performance of the first thermographic algorithm at different cut-off levels is presented. The optimal point on the ROC curve for the new algorithm (not shown), where the sensitivity and specificity were highest, corresponded to a cut-off level of 0.28 . Predicted probabilities above and below this cut-off would result in a confirmation or rejection of the diagnosis, respectively. This cut-off level yielded a sensitivity and specificity of $83 \%$ and $79 \%$, respectively. The PPV and NPV were $67 \%$ and $90 \%$, respectively. The accuracy was $80 \%$. However, the new algorithm should be tested in future patients in need of verification.

\section{Discussion}

The thermographic method and resulting algorithm for verifying RP, which was previously described [8], was tested in patients suspected of having RP. When applying a cut-off level lower than the cut-off level originally chosen, the diagnostic ability of the thermographic algorithm was noninferior to that of the FSP test. In addition, the thermographic method provided more information, was less time-consuming, and seemed more agreeable to the patient than the FSP test. Furthermore, a new thermographic algorithm was proposed.

A discussion relevant to many diagnostic procedures is the choice of cut-off level. An appropriate cut-off level depends on the diagnosis in question and the clinical consequence. Is it important to identify all patients with RP, or is it clinically more useful to exclude the patients without RP? This should be discussed with the physicians who rely on this method. The sensitivity of the thermographic method when tested on the patients suspected of RP with the proposed predicted probability cut-off point of 0.05 was $65 \%$, and the specificity was $58 \%$. The PPV was $77 \%$, and the NPV $44 \%$. The same values for the FSP test were $54 \%$, $58 \%, 74 \%$, and $37 \%$, respectively. The accuracy was $63 \%$ for the thermographic method and $55 \%$ for the FSP test. This indicates a similar diagnostic accuracy, where the strength of the thermographic method is the ability to identify patients with RP, and the physician can have confidence in a positive diagnosis.

In the literature, sensitivity and specificity for the FSP test ranged from $74 \%$ to $92 \%$ and $81 \%$ to $100 \%$, respectively, when the patients were tested against healthy controls. The new 
algorithm yielded a sensitivity and specificity of $83 \%$ and $79 \%$, respectively. Accordingly, the performance of the new algorithm — which has not yet been tested-seems to concur with the performance of the FSP test reported in the literature. The differences between studies evaluating the FSP method are likely due to differences in the study methods and population, such as the extent of cooling (temperature or local versus body cooling or both), the severity of RP in the examined patient group, and the vibration exposure level in the reference group. High sensitivities are usually found in studies applying both local and body cooling [13-15], low cooling temperatures [13-16], or high discriminating thresholds $(60-70 \%)$. In addition, studies have argued that the sensitivity increases with the disease severity $[16,17]$. The patients referred to our department for examination exhibit a wide range of RP symptoms, from patients with a few and nonspecific symptoms to patients with obvious symptoms of RP. Consequently, a lower sensitivity of any method than that reported in the literature should be expected. Although the patients' symptoms are diverse, the prevalence of RP and the symptom severity in the patients referred for examination do not seem to have changed markedly over the years of 2014-2018, based on the similar prevalence of RP in the retrospective and the prospective patient groups. As a result, there is reason to believe that the validated thermographic method and the proposed cut-off level will be useful for verifying RP in future patients referred for examination.

Compared with the previous study [8], where several parameters were significantly different on a group basis, only time to $t_{\text {end }}$ and curve type differed significantly between the confirmed patients and the controls in the present study. Most of the patients referred for examination and included in the present study were exposed to vibration and were suspected of having VWF. The considerable overlap between the patient and control groups for most of the predictors indicates that these patients are more difficult to distinguish from healthy individuals than patients with pRP. Furthermore, we found that the characteristics of $\mathrm{pRP}$ reported in the first algorithm differed from the characteristics of patients with $\mathrm{RP}$ mainly secondary to vibration exposure. This indicates that the two types of RP are two different disease entities with the same manifestations. In addition, the differences in the appropriate predictors for patients with pRP and VWF, respectively, confirm the findings of previous studies $[9-11,18]$. The curve type, $t_{50}$, and time to $t_{\text {end }}$ are all dynamic parameters. If the baseline parameters are considered a surrogate for tissue damage, as was indicated in references [5,11], these findings would suggest that VWF-at least, for the included patients-is a dynamic disease without considerable tissue damage to the vessels of the fingers. Although studies have shown inflammation and remodeling of the vessels and damage to the nerve endings with resulting vascular and sensorineural dysfunction after exposure to vibration $[19,20]$, our results are similar to the findings of von Bierbrauer et al. and House et al. $[9,10]$.

Thermal imaging has previously been deemed useful in the management of various diseases, such as breast cancer and diabetes [21]: in breast cancer, thermal imaging seems interesting as a detection tool; in diabetes, it has proven useful in the diagnosis of long-term sequelae, such as peripheral neuropathy $[22,23]$. Furthermore, thermal imaging is applied in rheumatology to distinguish patients with $\mathrm{pRP}$ from patients with systemic sclerosis [24]. In addition to being investigated in diagnostic settings, thermal imaging has been reported as a useful screening tool for large-scale fever assessments during pandemics to prevent the dispersion of the infection [25]. The possibility of automated readings of thermographic images by machine learning methods carries great potential. However, the use of machine learning requires large image databases [26], which are not necessarily available.

Although thermographic imaging has a high potential as a medical imaging method, various conditions during image acquisition can limit its use and accuracy. The thermographic reporting of the temperature of an object is highly dependent on the object's emissivity. Emissivity is the ability of the object to absorb and emit radiation compared to a theoretical "perfect emitter" with an emissivity of one. The emissivity of human skin is 0.98 [27], but several factors can affect its emissivity, which may result in the reporting of a lower temperature of the skin. Factors such as the presence of water [28], hair [29], or 
creams and lotions [28,29] on the skin can reduce its emissivity and, thereby, decrease the reported skin temperature. Theoretically, the degree of skin pigmentation might affect its emissivity, but skin color has been reported not to affect skin emissivity [27,29,30]. Accordingly, the participants in the present study wore thin plastic bags during cold provocation and were asked not to use lotions before the examination. Furthermore, we avoided placing regions of interest over the nails, as the emissivity of nails is unknown, and polished surfaces are known to have a lower emissivity than rough surfaces [31].

Along with the assessment of the test performances, other parameters such as patient discomfort, operability, and time consumption are of interest. In addition to generating more detailed information, the thermographic procedure was less technically demanding, less time-consuming, and seemed to be more pleasant for the patient than the FSP test. Indications of the patient content were based on the cooling method, time frame, and absence of episodes of RP during examination.

The patients of this study were patients referred to the department for examination. None refused participation or were excluded. Consequently, the results were highly generalizable to our department, as well as to other departments that examine unselected patients suspected of having RP mainly secondary to hand-arm vibration exposure and with similar broad symptom diversity. Furthermore, our study examined several parameters: both the baseline temperature, as well as dynamic parameters, after the cold challenge. The parameters of thermographic imaging often show considerable overlap between the patients and controls, and a combination of predictors seems necessary for diagnostic purposes.

Each participant was examined once, and test-retest reliability was not possible to assess from the current data, which poses a limitation. Although the reproducibility of dynamic thermographic imaging has been questioned, previous studies have shown good intraindividual reproducibility on both a group and an individual basis [18,32-34] Nevertheless, the test-retest reliability of the present thermographic method still requires investigation. Information bias poses another limitation; the level of detail of the medical records was diverse, possibly leading to an incorrect evaluation by the readers and, thereby, an inaccurate response variable. Ultimately, this could have affected the testing of the algorithm and the fitting of the new prediction model.

\section{Conclusions}

A recently developed thermographic algorithm for verifying RP was tested in patients who were suspected of having RP and who were part of the target population. The accuracy of the thermographic algorithm was noninferior to the that of the currently applied FSP test. In addition, the thermographic method seemed easier to operate, more informative, and more agreeable to the patient than the FSP procedure. An entirely new algorithm was proposed, and the new model revealed that the predictors of pRP differed from the predictors of VWF, indicating that the two types of RP are different diseases with similar manifestations. Although the new algorithm seems useful as a diagnostic tool, its diagnostic ability must be validated in a future patient population of similar composition before it can be put into practice. Meanwhile, the original thermographic algorithm tested in the present study can replace the FSP test as a method for verifying RP. Although it is reasonable for an objective examination to assist decision-making, the results of either verification method should never be able to overrule obvious clinical symptoms of RP.

Author Contributions: Conceptualization, L.L. and L.T.J.; methodology, L.L., B.K., J.F.T., E.E. and L.T.J.; software, L.L. and B.K.; validation, L.T.J.; formal analysis, L.L. and B.K.; investigation, L.L.; data curation, L.L.; writing-original draft preparation, L.L.; writing-review and editing, L.L., B.K., J.F.T., E.E. and L.T.J.; visualization, L.L. and B.K.; supervision, L.T.J.; and project administration, L.L. and L.T.J. All authors have read and agreed to the published version of the manuscript.

Funding: This research received no external funding. 
Institutional Review Board Statement: The study was conducted according to the guidelines of the Declaration of Helsinki and approved by the Research Ethics Committee of the Capital Region of Denmark (protocol code H-16035842, approved on 5 October 2016).

Informed Consent Statement: Informed consent was obtained from all subjects involved in the study.

Data Availability Statement: The data presented in this study are available on request from the corresponding author.

Conflicts of Interest: The authors declare no conflict of interest.

\section{References}

1. Herrick, A.L. The pathogenesis, diagnosis and treatment of Raynaud phenomenon. Nat. Rev. Rheumatol. 2012, 8, 469-479. [CrossRef] [PubMed]

2. Aarhus, L.; Stranden, E.; Nordby, K.C.; Einarsdottir, E.; Olsen, R.; Ruud, B.; Bast-Pettersen, R. Vascular component of hand-arm vibration syndrome: A 22-year follow-up study. Occup. Med. 2018, 68, 384-390. [CrossRef] [PubMed]

3. Nielsen, S.L. Raynaud phenomena and finger systolic pressure during cooling. Scand. J. Clin. Lab. Investig. 1978, 38, 765-770. [CrossRef] [PubMed]

4. Coughlin, P.A.; Chetter, I.C.; Kent, P.J.; Kester, R.C. The analysis of sensitivity, specificity, positive predictive value and negative predictive value of cold provocation thermography in the objective diagnosis of the hand-arm vibration syndrome. Occup. Med. 2001, 51, 75-80. [CrossRef] [PubMed]

5. Schuhfried, O.; Vacariu, G.; Lang, T.; Korpan, M.; Kiener, H.P.; Fialka-Moser, V. Thermographic parameters in the diagnosis of secondary Raynaud's phenomenon. Arch. Phys. Med. Rehabil. 2000, 81, 495-499. [CrossRef]

6. Herrick, A.L.; Clark, S. Quantifying digital vascular disease in patients with primary Raynaud's phenomenon and systemic sclerosis. Ann. Rheum. Dis. 1998, 57, 70-78. [CrossRef]

7. Ye, Y.; Griffin, M.J. Effect of room temperature on tests for diagnosing vibration-induced white finger: Finger rewarming times and finger systolic blood pressures. Int. Arch. Occup. Environ. Health 2017, 90, 527-538. [CrossRef]

8. Lindberg, L.; Kristensen, B.; Thomsen, J.F.; Eldrup, E.; Jensen, L.T. Characteristic Features of Infrared Thermographic Imaging in Primary Raynaud's Phenomenon. Diagnostics 2021, 11, 558. [CrossRef]

9. House, R.; Holness, L.; Taraschuk, I.; Nisenbaum, R. Infrared thermography in the hands and feet of hand-arm vibration syndrome (HAVS) cases and controls. Int. J. Ind. Ergon. 2017, 62, 70-76. [CrossRef]

10. Von Bierbrauer, A.; Schilk, I.; Lucke, C.; Schmidt, J.A. Infrared thermography in the diagnosis of Raynaud's phenomenon in vibration-induced white finger. VASA Z. Gefasskrankh. 1998, 27, 94-99.

11. Cherkas, L.F.; Carter, L.; Spector, T.D.; Howell, K.J.; Black, C.M.; MacGregor, A.J. Use of thermographic criteria to identify Raynaud's phenomenon in a population setting. J. Rheumatol. 2003, 30, 720-722.

12. O'Reilly, D.; Taylor, L.; El-Hadidy, K.; Jayson, M.I.V. Measurement of cold challenge responses in primary Raynaud's phenomenon and Raynaud's phenomenon associated with systemic sclerosis. Ann. Rheum. Dis. 1992, 51, 1193-1196. [CrossRef]

13. Olsen, N.; Nielsen, S.L. Diagnosis of Raynaud's phenomenon in quarrymen's traumatic vasospastic disease. Scand. J. Work Environ. Health 1979, 5, 249-256. [CrossRef] [PubMed]

14. Olsen, N.; Nielsen, S.L.; Voss, P. Cold response of digital arteries in chain saw operators. Br. J. Ind. Med. 1982, 39, 82-88. [CrossRef] [PubMed]

15. Olsen, N. Diagnostic tests in Raynaud's phenomena in workers exposed to vibration: A comparative study. Br. J. Ind. Med. 1988, 45, 426-430. [CrossRef] [PubMed]

16. Pyykko, I.; Farkkila, M.; Korhonen, O.; Starck, J.; Jäntti, V. Cold provocation tests in the evaluation of vibration-induced white finger. Scand. J. Work Environ. Health 1986, 12, 254-258. [CrossRef] [PubMed]

17. Ekenvall, L.; Lindblad, L.E. Vibration white finger and digital systolic pressure during cooling. Br. J. Ind. Med. 1986, 43, 280-283. [CrossRef] [PubMed]

18. Lim, M.J.; Kwon, S.R.; Jung, K.; Joo, K.; Park, S.; Park, W. Digital Thermography of the Fingers and Toes in Raynaud's Phenomenon. J. Korean Med. Sci. 2014, 29, 502-506. [CrossRef] [PubMed]

19. Krajnak, K.; Riley, D.A.; Wu, J.; McDowell, T.; Welcome, D.E.; Xu, X.S.; Dong, R.G. Frequency-dependent effects of vibration on physiological systems: Experiments with animals and other human surrogates. Ind. Health 2012, 50, 343-353. [CrossRef]

20. Stoyneva, Z.; Lyapina, M.; Tzvetkov, D.; Vodenicharov, E. Current pathophysiological views on vibration-induced Raynaud's phenomenon. Cardiovasc. Res. 2003, 57, 615-624. [CrossRef]

21. Lahiri, B.B.; Bagavathiappan, S.; Jayakumar, T.; Philip, J. Medical applications of infrared thermography: A review. Infrared Phys. Technol. 2012, 55, 221-235. [CrossRef]

22. Sun, P.C.; Lin, H.D.; Jao, S.H.E.; Ku, Y.C.; Chan, R.C.; Cheng, C.K. Relationship of skin temperature to sympathetic dysfunction in diabetic at-risk feet. Diabetes Res. Clin. Pract. 2006, 73, 41-46. [CrossRef]

23. Lavery, L.A.; Higgins, K.R.; Lanctot, D.R.; Constantinides, G.P.; Zamorano, R.G.; Athanasiou, K.A.; Armstrong, D.G.; Agrawal, C.M. Preventing diabetic foot ulcer recurrence in high-risk patients: Use of temperature monitoring as a self-assessment tool. Diabetes Care 2007, 30, 14-20. [CrossRef] 
24. Anderson, M.E.; Moore, T.L.; Lunt, M.; Herrick, A.L. The "distal-dorsal difference": A thermographic parameter by which to differentiate between primary and secondary Raynaud's phenomenon. Rheumatology 2007, 46, 533-538. [CrossRef]

25. Perpetuini, D.; Filippini, C.; Cardone, D.; Merla, A. An overview of thermal infrared imaging-based screenings during pandemic emergencies. Int. J. Environ. Res. Public Health 2021, 18, 1-12. [CrossRef]

26. Shaikh, F.; Dehmeshki, J.; Bisdas, S.; Roettger-Dupont, D.; Kubassova, O.; Aziz, M.; Awan, O. Artificial Intelligence-Based Clinical Decision Support Systems Using Advanced Medical Imaging and Radiomics. Curr. Probl. Diagn. Radiol. 2021, 50, $262-267$. [CrossRef] [PubMed]

27. Charlton, M.; Stanley, S.A.; Whitman, Z.; Wenn, V.; Coats, T.J.; Sims, M.; Thompson, J.P. The effect of constitutive pigmentation on the measured emissivity of human skin. PLoS ONE 2020, 15, e0241843. [CrossRef] [PubMed]

28. Bernard, V.; Staffa, E.; Mornstein, V.; Bourek, A. Infrared camera assessment of skin surface temperature-Effect of emissivity. Phys. Med. 2013, 29, 583-591. [CrossRef]

29. Fernández-Cuevas, I.; Bouzas Marins, J.C.; Arnáiz Lastras, J.; Gómez Carmona, P.M.; Piñonosa Cano, S.; García-Concepción, M.Á.; Sillero-Quintana, M. Classification of factors influencing the use of infrared thermography in humans: A review. Infrared Phys. Technol. 2015, 71, 28-55. [CrossRef]

30. Steketee, J. Spectral emissivity of skin and pericardium. Phys. Med. Biol. 1973, 18, 686-694. [CrossRef] [PubMed]

31. Wen, C.D.; Mudawar, I. Modeling the effects of surface roughness on the emissivity of aluminum alloys. Int. J. Heat Mass Transf. 2006, 49, 4279-4289. [CrossRef]

32. Cleophas, T.J.M.; Fennis, J.F.M.; Van't Laar, A. Finger temperature after a finger-cooling test: Influence of air temperature and smoking. J. Appl. Physiol. 1982, 52, 1167-1171. [CrossRef] [PubMed]

33. Wilkinson, J.D.; Leggett, S.A.; Marjanovic, E.J.; Moore, T.L.; Anderson, M.E.; Britton, J.; Buch, M.H.; Del Galdo, F.; Denton, C.P.; Dinsdale, G.; et al. A Multicenter Study of the Validity and Reliability of Responses to Hand Cold Challenge as Measured by Laser Speckle Contrast Imaging and Thermography Outcome Measures for Systemic Sclerosis—Related Raynaud's Phenomenon. Arthritis Rheumatol. 2018, 70, 903-911. [CrossRef] [PubMed]

34. Pauling, J.D.; Shipley, J.A.; Harris, N.D.; McHugh, N.J. Use of infrared thermography as an endpoint in therapeutic trials of Raynaud's phenomenon and systemic sclerosis. Clin. Exp. Rheumatol. 2012, 30, 103-115. 